
by consonant music and facilitated by

SUBJECT AREAS:

LANGUAGE

ATTENTION

COGNITIVE AGEING

EVOLUTION OF LANGUAGE

Received

14 March 2013

Accepted

3 June 2013

Published

19 June 2013

Correspondence and requests for materials should be addressed to

N.M. (masataka. nobuo.7r@kyoto-u.ac. \section{dissonant music}

\author{
Nobuo Masataka' \& Leonid Perlovsky²
}

\begin{abstract}
${ }^{1}$ Primate Research Institute, Kyoto University, Kanrin, Inuyama, Aichi, Japan, ${ }^{2}$ Athinoula A. Martinos Center for Biomedical Imaging, Harvard University, Charlestown, MA, USA and Air Force Research Laboratory, Dayton, OH, USA.
\end{abstract}

Debates on the origins of consonance and dissonance in music have a long history. While some scientists argue that consonance judgments are an acquired competence based on exposure to the musical-system-specific knowledge of a particular culture, others favor a biological explanation for the observed preference for consonance. Here we provide experimental confirmation that this preference plays an adaptive role in human cognition: it reduces cognitive interference. The results of our experiment reveal that exposure to a Mozart minuet mitigates interference, whereas, conversely, when the music is modified to consist of mostly dissonant intervals the interference effect is intensified.

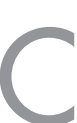

ontemporary cognitive and evolutionary musicology faces great controversies in attempting to identify the cognitive function of music and its evolutionary origins ${ }^{1-7}$. Arguments about the power of music over human psyche began long ago. Aristotle ${ }^{8}$ listed the power of music among the unsolved problems alongside finiteness of the world and existence of God. Kant ${ }^{9}$ was able to explain the epistemology of the beautiful by relating it to knowledge, but could not explain music: "it merely plays with senses." Darwin ${ }^{10}$ noted that the human ability for music "must be ranked amongst the most mysterious with which (man) is endowed" because music is a human cultural universal that appears to serve no obvious adaptive purpose.

Among current evolutionary psychologists and musicologists, some consider that music plays no adaptive role in human evolution. So following Kant, Pinker ${ }^{11}$ has argued that music is an "auditory cheesecake," a byproduct of natural selection that just happened to "tickle the sensitive spots." While other contemporary scientists ${ }^{12,13}$ suggested that music clearly has an evolutionary role, pointed to music's universality, and continued developing Darwin's idea on sexual selection of music, a review ${ }^{14}$ about kin selection and other biological mechanisms for music evolution concluded that "no one selective force (e.g., sexual selection) is adequate to explaining all aspects of human music." In 2008, Nature published a series of essays on music ${ }^{5}$, the authors however could not agree on the evolutionary origin or cognitive function of music.

Meanwhile, potential cognitive benefits of music have been explored in the context of the so called "Mozart effect ${ }^{1 "}$. This is a short-term improvement on "spatial-temporal reasoning." The finding has been hyped by the media so that many scientists conducted experiments to verify its validity. A short-term effect of any improvement was illustrated though specificity to Mozart and music was questioned ${ }^{15,16}$. Subsequently, an experimental brain activation study demonstrated that the "Mozart effect" can be accounted for by controlling for mood and arousal $^{17}$.

Recently we have presented experimental evidence ${ }^{18,19}$ supporting a hypothesis that music has a fundamental cognitive function: to help to mitigate cognitive dissonance. Cognitive dissonance is a discomfort caused by holding conflicting cognitions simultaneously ${ }^{20}$. It usually leads to devaluation and discarding of conflicting knowledge $^{21,22}$. This theory is among the most influential and extensively studied theories in psychology. It is intimately connected to the entirety of human evolution. At the dawn of human evolution, the emergence of language led to the proliferation of cognitive dissonances ${ }^{23,24}$. If they had not been overcome, language and knowledge would have been discarded and further human evolution would have been stopped in its tracks. This is why the ability of music to mitigate cognitive dissonance could be fundamental for musical cognitive function and music evolution ${ }^{25}$. Our attempts to experimentally confirm this hypothesis were undertaken using a classical paradigm ${ }^{26}$ known to create cognitive dissonance. Exposure to Mozart's music enabled participants to overcome the cognitive dissonance. This tentatively supports the argument that music has a fundamental cognitive function, which defines its evolutionary role and leads to music's universality ${ }^{18-21}$. Additional 
experimental evidence ${ }^{27}$ confirmed that music can help overcoming cognitive dissonance in the context of academic tests. The present study was conducted to pursue this issue further.

Here we evaluated the effects of music on cognitive interference. To create cognitive interference, we used a prototypical task known as the "Stroop interference task ${ }^{28 "}$ ", which requires a person to respond to a specific dimension of a stimulus while suppressing a competing stimulus dimension. In the task, typically, a colour word such as GREEN appears in an ink colour such as red. If the participant's task is to read the word and ignore the colour (e.g. say "green"), there is no evidence of difficulty reading the word compared to reading it when printed in standard black ink. However, if the participant's task is to name the ink colour and ignore the word (e.g. say "red"), there is considerable difficulty relative to reading a colour patch. Reading the word interferes with naming the colour, but the colour does not interfere with reading the word. This is the phenomenon of Stroop interference.

We have conducted the present experiment on the basis of the hypothesis that the magnitude of such cognitive interference would be reduced when a person is tested with exposure to more consonant intervals than dissonant intervals. Our hypotheses have been, first, that music with more consonant intervals would reduce cognitive interference relative to that when the same person was tested without exposure to any music. Our second hypothesis has been that music with more dissonant intervals would increase cognitive interference relative to that when the same person was tested without exposure to any music.

We used a modified version of Stroop interference task known as the colour-word matching Stroop task ${ }^{29}$. Children aged 8- to 9-yearsold and elderly adults aged 65 to 75 years old participated in the present experiment. They were asked to name the ink of a colour word that designated a colour incongruent with that of the ink of the word ('Incongruent test session'). Also, the same participants were tested in a 'Neutral testing session', in which they were asked to name the ink of a colour of a non-word string of letters, i.e., XXX. Both sessions were repeated under three conditions: (1) with exposure to music containing predominantly consonant intervals (Consonant condition), (2) with exposure to music containing predominantly dissonant intervals (Dissonant condition), and (3) without exposure to any music (Control condition). In every session and condition, the performance of the participants was measured as reaction time (RT) to response and error rate (ER) of the response.
The consonant music used was the original version of one of Mozart's minuets, most of which consisted of consonant intervals. For dissonant music we used a modified version of the minuet, most of which consisted of dissonant intervals. The same auditory stimuli were used in previous research ${ }^{30-32}$ that revealed perceptual preferences for consonance over dissonance in music in young infants and newborns.

\section{Results}

Figure 1 shows the performance results of the children assessed using RT. These results were analyzed using repeated measures of analysis of variance (ANOVA) with the following two factors: Incongruent vs. Neutral test sessions, and Consonant or Dissonant vs. Control conditions. This analysis demonstrated a significant main effect for test (Incongruent vs. Neutral test sessions, F(1,24) $=407.02, \mathrm{P}<$ 0.001). The main effect of condition was also significant (Consonant or Dissonant vs. Control conditions, $\mathrm{F}(2,48)=58.59, \mathrm{P}<0.001)$. There was a significant interaction between the two factors $(F(2,48)$ $=3.69, \mathrm{P}=0.024)$. Post-hoc comparisons using Tukey's Honestly Significant Difference (HSD) tests revealed that under each of the three conditions, RTs were significantly longer for Incongruent session vs. Neutral session (Ps $<0.001$ ). Moreover, the mean RTs for Incongruent sessions were significantly shorter under the Consonant condition than under the Dissonant or Control conditions (Ps $<$ 0.001 ), confirming our hypothesis. Similarly, the RT under the Dissonant condition was significantly longer than that under the Control condition $(\mathrm{P}=0.032)$. On the other hand, in Neutral sessions, the mean RTs did not differ among the three conditions (Ps $>$ 0.711). All these results confirmed our expectations: the Stroop effect results in cognitive interference as expected, and our hypotheses were confirmed: consonant music helps to overcome cognitive interference, and dissonant music increases the interference.

The results obtained using ER in the children are presented in Figure 2, and are similar to the results obtained using RT, confirming the effects seen above. There was a significant main effect for test $(\mathrm{F}(1,24)=480.89, \mathrm{P}<0.001)$. Main effect of condition was also significant $(\mathrm{F}(2,48)=67.46, \mathrm{P}<0.001)$. There was a significant interaction between the two factors $(\mathrm{F}(2,48)=24.45, \mathrm{P}<0.001)$. Under each of the three conditions, ERs recorded from the participants were significantly greater during Incongruent sessions than during Neutral sessions (Ps $<0.001)$. The mean score for Incongruent sessions was significantly smaller under Consonant

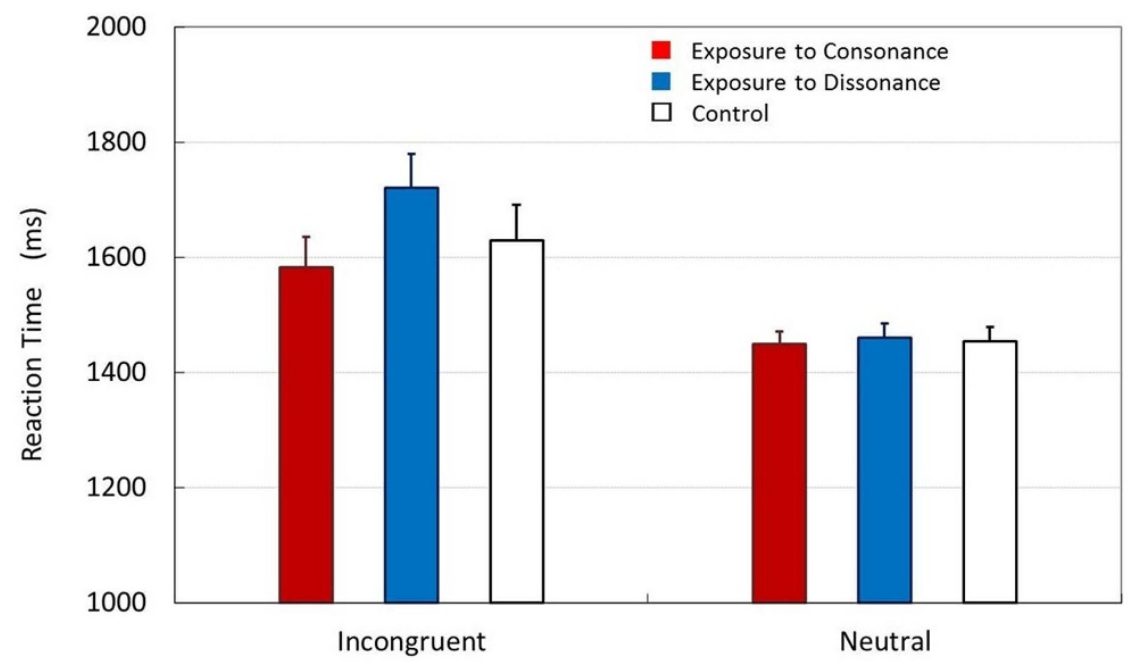

Figure 1 Experiments. Mean reaction time (RTs) of the participantchildren for the Stroop task (in 'Incongruent' sessions) with exposure to the original (consonant) version of one of Mozart's minuets (Consonant condition; Exposure to Consonance), the modified (dissonant) version of the minuet (Dissonant condition; Exposure to Dissonance); and without exposure to any music (Control), and RTs of the participants under these three conditions in 'Neutral' testing sessions. Error bars represent SDs. 


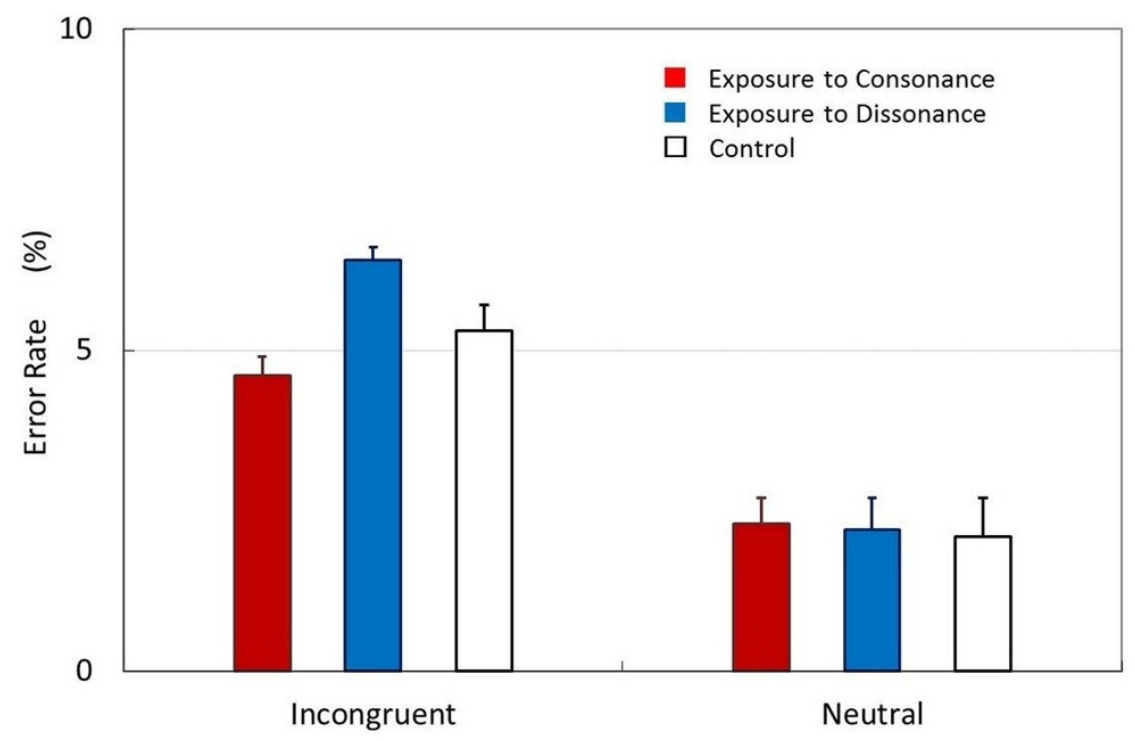

Figure $2 \mid$ Experiments. Mean error rate (ERs) of the participant children for the Stroop task (in the 'Incongruent' testing session) with exposure to the original (consonant) version of a Mozart minuet (Consonance condition; Exposure to Consonance), with exposure to the modified (dissonant) version of the minuet (Dissonant condition; Exposure to Dissonance), and without exposure to any music (Control), and ERs of the participants under the three conditions in the 'Neutral' testing session. Error bars represent SDs.

condition than under Dissonant condition or Control condition (Ps $<0.001)$. The score under Dissonant condition was significantly greater than that under Control condition, too $(P=0.028)$. For Neutral sessions, the mean ERs did not differ among the three tests (Ps $>0.752)$. All of these results give additional confirmation of our hypotheses.

The results of the experiment with the elderly adults were strikingly similar to those with the children. Figure 3 illustrates the performance results using RT. Significant main effects were found for test (Incongruent vs. Neutral test sessions, $\mathrm{F}(1,24)=465.93, \mathrm{P}<$ 0.001 ) as well as for condition (Consonant or Dissonant vs. Control conditions, $\mathrm{F}(2,48)=73.08, \mathrm{P}<0.001)$. There was also a significant interaction between the two factors $(\mathrm{F}(2,48)=29.59, \mathrm{P}<0.001)$. Under each of the three conditions RTs were significantly greater for Incongruent session vs. Neutral session (Ps $<0.001$ ). Moreover, the mean scores for Incongruent session were significantly smaller under
Consonant condition than under Dissonant or Control conditions (Ps $<0.001)$. The score under Dissonant condition was significantly greater than that under Control condition $(\mathrm{P}=0.017)$. In contrast, in Neutral sessions the mean RTs did not differ among the three conditions (Ps $>0.841$ ).

The results for ER in the elderly adults are presented in Figure 4. There was a significant main effect for test $(\mathrm{F}(1,24)=510.47, \mathrm{P}<$ $0.001)$. The main effect of condition was also significant $(\mathrm{F}(2,48)=$ $82.49, \mathrm{P}<0.001)$. There was a significant interaction between the two factors $(\mathrm{F}(2,48)=27.65, \mathrm{P}<0.001)$. Under each of the three conditions, ERs recorded for the participants were significantly greater during Incongruent sessions than during Neutral sessions (Ps $<0.001)$. The mean score for Incongruent sessions was significantly smaller under Consonant condition than under Dissonant condition or Control condition (Ps $<0.001)$. The score under Dissonant condition was significantly greater than that under

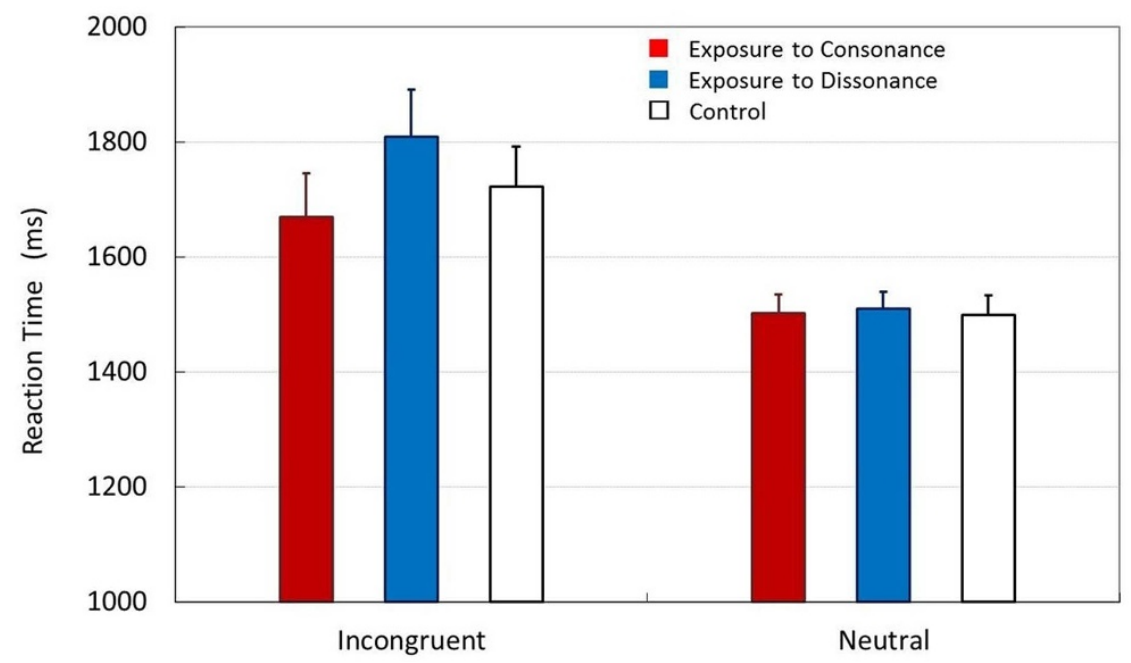

Figure 3 Experiments. Mean reaction time (RTs) of the participant elderly adults for the Stroop task (in 'Incongruent' sessions) with exposure to the original (consonant) version of a Mozart's minuet (Consonant condition; Exposure to Consonance), the modified (dissonant) version of the minuet (Dissonant condition; Exposure to Dissonance); and without exposure to any music (Control), and RTs of the participants under the three conditions in 'Neutral' testing session. Error bars represent SDs. 


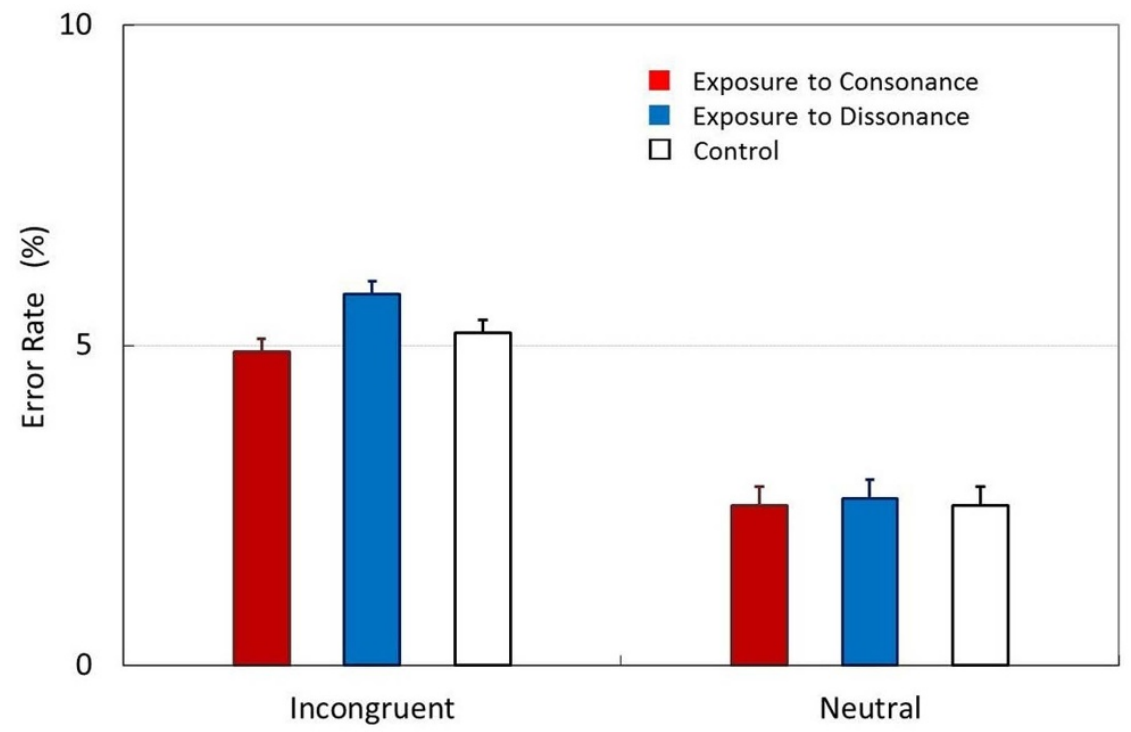

Figure 4 Experiments. Mean error rate (ERs) of the participant elderly adults for the Stroop task (in 'Incongruent' testing session) with exposure to the original (consonant) version of a Mozart's minuet (Consonance condition; Exposure to Consonance), with exposure to the modified (dissonant) version of the minuet (Dissonant condition; Exposure to Dissonance), and without exposure to any music (Control), and ERs of the participants under the three conditions in 'Neutral' testing session. Error bars represent SDs.

Control condition, too $(\mathrm{P}=0.022)$. For Neutral sessions the mean ERs did not differ among the three tests (Ps $>0.863$ ).

\section{Discussion}

As reported previously ${ }^{29,33,34}$, the colour-word matching Stroop task produced an expected interference effect in the present experiment, which remained significant during the entire test. The observed results are similar to traditionally reported Stroop effects, and the main sources of difficulty are similar: cognitive interference. The new result corresponding to our hypothesis is that the magnitude of such interference was reduced when the participants were exposed to a Mozart minuet with primarily consonant intervals, and the interference was increased when the participants were exposed to a modified minuet with primarily dissonant intervals. We conclude that consonant music may have an important cognitive function: help overcoming cognitive interference. Together with other recent experimental and theoretical publication $s^{18,19,23-25}$ this gives tentative support for our hypothesis about the fundamental cognitive function of music: it helps to resolve cognitive interference, cognitive dissonance, and facilitates human evolution.

Another issue addressed in this paper is the role of consonant vs. dissonant music and their relations to pleasure of music. It is known that infants and even newborns exhibit strong perceptual preferences for the original minuet containing mostly consonant intervals over its modified dissonant version ${ }^{29-31,35}$. Consonant intervals are prevalent in most works of music ${ }^{36,37}$. Our results therefore support recent findings that the effect of music on cognitive dissonance depends on the hedonicity of music: pleasant music better helps to overcome cognitive dissonance than unpleasant music ${ }^{38}$.

Drawing conclusions about the connection between musical consonance and hedonicity requires caution since dissonant and sad music could also be sources of pleasure. Now we will discuss possible cognitive functions of musical dissonance, an unresolved issue in psychology and musicology $y^{35-37}$. We demonstrated that the modified version of the minuet containing more musical dissonance increased the cognitive interference. This result might be related to the findings of a recent non-invasive brain activation study ${ }^{17}$ whose aim was to debunk the popular version of the 'Mozart effect' and to demonstrate that any improvement on cognitive tests after listening to Mozart is not specific to music. Those authors reported that whereas Mozart's music results in some improvements of cognitive test scores, Adagio by Albinoni (sad, slow music) results in lower scores on the same cognitive tests; they demonstrated that mood and arousal may account for the 'Mozart effect,' but did not address why the Albinoni adagio is among the most popular pieces of music.

So what could be the cognitive function of musical dissonance and of music per se and evolutionary reasons for music evolution? Our original hypothesis suggested that one cognitive function of music is to overcome a large number of cognitive dissonances between virtually any two cognitions ${ }^{24,38}$. These include stress that arises in many complicated and difficult life conditions much more difficult and trying than those evoked by the Stroop effect.

A specific aspect of this question is why sad music is pleasurable ${ }^{39}$. One of the most popular pieces of western classical music is Adagio by Barber, which is sad, slow, and highly dissonant, like Adagio by Albinoni. According to our hypothesis ${ }^{19,23-25,38}$, sad music helps to overcome dissonance arising from difficult life conditions, including the ultimately death of close people (the dissonance between the feeling of the infinity of the spirit and the knowledge of death). In general, any two (or more) cognitions involve a cognitive dissonance $e^{24,25,38}$. Possibly, the cognitive dissonance between any two cognitions involves its own shade of emotion, and overcoming each cognitive dissonance requires a special musical emotion (we do not differentiate here between emotion and $\operatorname{mood}^{39}$ ). This hypothesis implies a potentially large number of musical emotions and also of cognitive dissonances and interferences. Music evolved for helping to overcome the predicament of stress that arises from holding contradictory cognitions, so that knowledge is not discarded, but rather can be accumulated, and human culture can evolve $^{25}$. Our experimental results ${ }^{18,19}$ emphasize a need for further research studying multiple emotions and for determining the dimensionality of these emotional spaces. This problem has not been solved, and the current paper reports a step in this direction. The consonance-dissonance dimension explored here is related to hedonicity (pleasure or displeasure) perceived in music ${ }^{38}$; however, the potential pleasure from sad dissonant music makes this connection nontrivial. Possibly music is perceived as pleasant if it resolves cognitive dissonances and interferences important for a listener. Music pleasant for many people resolves dissonances and interferences important for many of us. 
While the non-invasive brain activation study mentioned above ${ }^{17}$ postulated that the "Mozart effect" is equivalent to mood and arousal, we have not explored differences between moods and emotions here mainly because we hypothesize, as discussed above, relationships between musical emotions and cognitive dissonances should be much more complicated than that ${ }^{17}$ had been assumed without addressing a fundamental question of why the Adagio by Albinoni is among most popular pieces of western music. In view of our hypothesis about relationships between musical emotions and cognitive dissonances, differences among emotions and mood are certainly worth discussing to allow an understanding the differences and similarities between (various) work in this field. Meanwhile we admit that the issue remains a part of much more complicated problem that would have to be explored in future research.

The Stroop task is well known as an effective test for examining the cognitive processes of inhibition and interference resolution ${ }^{40}$. In this test, interference occurs at the conceptual level and is separated from the response preparation. Performance in the test continues to develop up to approximately 17-19 years of age, and thereafter is likely to decline gradually as aging proceeds ${ }^{41,42}$. This developmental pattern of changes likely reflects underlying changes in the brain, which are profoundly related to executive function and prefrontal brain activation ${ }^{40,42,43}$. The current study presents experimental evidence for the modulation of executive function by music. It seems that consonant music enhances the inhibition function of the executive control while dissonant music might exert a disinhibition influence "emancipating" the person from the control of the executive function. Testing this hypothesis would also require further research.

\section{Methods}

This investigation was conducted according to the principles expressed in the Declaration of Helsinki. All experimental protocols are consistent with the Guide for the Experimentation with Humans and were approved by the Institutional Ethical Committee of Primate Research Institute, Kyoto University.

Participants. As participants, we recruited 25 typically developing healthy 8 - to 9 year-old boys from several elementary schools and 25 healthy 65- to 75-year-old elderly adults from temporary employment agencies in Kyoto and Aichi prefectures, Japan. All were right-handed and had been exposed to the Japanese language as their first language. They were not using any medication that would influence performance on the experimental task. Normal cognitive status was verified in the elderly adults through prescreening at the time of evaluation (Mini-Mental State Examination) ${ }^{44,45}$ We obtained written informed consent from the parents of each of the participant children as well as from each of the participant elderly adults involved in our study. The experimental room was a sound-attenuated playroom $(3.5 \mathrm{~m} \times 5.5 \mathrm{~m})$ familiar to all of the participants. It contained a one-way observation mirror, a chair and a table. A 22-inch monitor connected with a personal computer was placed on the table. A ceiling speaker connected with an audio player was installed in the ceiling of the room, just above the table.

Procedure. An adapted single trial version of the colour-word matching Stroop task ${ }^{29,33,34}$ was used in the present experiment. Participants were told by an experimenter, who had not been notified about the purpose of the present experiment, that they would see two rows of letters appear on the screen of the monitor on the table and were instructed to decide, via button-press, if the colour of the top row letters corresponded to the colour name written at the bottom row. The index (YES-response) and middle finger (NO-response) of the right hand were used to respond. During trials in "Neutral testing sessions", the letters in the top row were "XXX" printed in red, green, blue, or yellow, and the bottom row consisted of the colour words "RED," "GREEN," "BLUE," and "YELLOW" printed in the Japanese language in black. For trials in "Incongruent testing sessions", the top row consisted of the colour words "RED," "GREEN," "BLUE," and "YELLOW" printed in the Japanese language in an incongruent colour (e.g., "green" printed in red) in order to produce an interference between the colour word and colour name. In order to prevent participants from focusing on the lower word and blurring out the top word, the top word was presented $100 \mathrm{~ms}$ before the lower word. By this, visual attention is shifted automatically to the top word. The participants decided in all conditions if the colour name of the top row corresponded with the colour word of the bottom row. The meaning of letters or words (e.g., "XXX" or "GREEN") was task irrelevant.

A testing session, whether a Neutral one or an Incongruent one, consisted of 40 trials in random order with an interstimulus interval of $12 \mathrm{~s}$. In half of the 40 trials in an Incongruent testing session, the colour of the top row letters was congruent with the colour name written at the bottom row, and both were incongruent with one another in the other half of the trials. We excluded congruent trials from the analysis as in previous research ${ }^{40}$.
In all, each participant was subjected to a Neutral testing session and to an Incongruent testing session, respectively, three times; once with exposure to the original version of Mozart's minuet (Consonant condition), once with exposure to the modified version of the minuet (Dissonant condition), and once without exposure to any music (Control condition). The order of the total 6 sessions was randomized. Each testing session was conducted for each participant with an interval of 7 days. In each testing session, the experimenter led each participant into the experimental room, closed the door and remained together with the participant until the end of the session. When the participant was subjected to the test under Consonant condition or Dissonant condition, the experimenter switched on the audio player as she was entering the room so that the original version or the modified version of the minuet was played, respectively (the sound pressure level: $65 \mathrm{~dB}$ ). The music continued to be played repeatedly until the experimenter switched off the player as the testing session finished.

The minuet was a simple one in $\mathrm{C}$ major by Mozart, $\mathrm{K}$. \# 1f. It was essentially the same as used previously ${ }^{31,32,33}$. Both the original and the modified versions were digitally generated and created by piano timbre. They were made up of 60 intervals. In the original version, only three of them were dissonant, and all three were tritons (6semitone intervals). In the modified version, all Gs were changed to F\#s and all Ds to C\#s. This had the effect of creating 21 additional dissonant intervals, including a total of 12 of the two most-dissonant intervals, i.e. seven tritons and five minor ninths (13 semitones). In the present stimuli, the upper voice and the lower voice were separated by more than an octave in each interval. The tempo was identical across the two versions (120 quarter per min).

1. Rauscher, F. H., Shaw, G. L. \& Ky, K. N. Music and spatial task performance Nature 365, 611 (1993).

2. Cooper, J. S. The Mozart effect. J Royal Soc Med 94, 170-172 (2001).

3. Cross, I. Music and cognitive evolution. In R. Dunbar \& L. Barrett (Eds.) Oxford handbook of evolutionary psychology, pp.649-667, (Oxford University Press, 2007).

4. Ball, P. Facing the music. Nature 453, 160-162 (2008)

5. Editorial. Bountiful noise. Nature 453, 134 (2008).

6. Masataka N. The origins of language (Springer, 2008).

7. Masataka $\mathrm{N}$. The origins of language and the evolution of music: a comparative study. Phys Life Rev 6, 11-22 (2009).

8. Aristotle. The complete works: the revised Oxford translation (Princeton University Press, 1995).

9. Kant, I. Kritik der Urteilskraft (Leipzig F Meiner, 1790).

10. Darwin, C. R. The descent of man, and selection in relation to sex (John Murray, 1871).

11. Pinker, S. How the mind works (Norton; 1997).

12. Masataka N. The onset of language (Cambridge University Press, 2003).

13. Miller, G. F. How did language evolve. In H. Swain (Ed.) Big questions in science, pp. 79-90, (Jonathan Cape, 2003).

14. Fitch, W. T. The biology and evolution of music: a comparative perspective. Cognition 100, 173-215 (2006).

15. Steele, K. M. et al. Prelude or requiem for the 'Mozart effect'? Nature 400, 827 (1999).

16. Schellenberg, E. G. Exposure to music: the truth about the consequences. In G. E. McPherson (Eds.) The child as musician: a handbook of musical development, pp. 111-134, (Oxford University Press, 2006).

17. Thompson, W. F., Schellenberg, E. G. \& Husain, G. Arousal mood and the Mozart effect. Psych Sci 12, 248-51 (2001).

18. Masataka, N. \& Perlovsky, L. I. Music can reduce cognitive dissonance. Nature Prec hdl:10101/npre.2012.7080.1 (2012)

19. Masataka, N. \& Perlovsky, L. I. The efficacy of musical emotions provoked by Mozart's music for the reconciliation of cognitive dissonance. Sci Rep 2, 307 (2012b).

20. Wikipedia. Cognitive dissonance. http://en.wikipedia.org/wiki/ Cognitive_dissonance (February 26th, 2013).

21. Cooper, J. Cognitive dissonance: 50 years of a classic theory (Sage, 2007).

22. Festinger, L. A theory of cognitive dissonance (Stanford University Press, 1957).

23. Perlovsky, L. I. Musical emotions: functions, origin, evolution. Phys Life Rev 7, 2-27 (2010).

24. Perlovsky, L. I. Cognitive function of music, part I. Interdisc Sci Rev 37, 129-42 (2012).

25. Perlovsky, L. I. A challenge to human evolution - cognitive dissonance. Front Psychol 4,179 (2013).

26. Aronson, E \& Carlsmith, J. M. Effect of the severity of threat on the devaluation of forbidden behavior. J Abnor Soc Psych 66, 584-588 (1963).

27. Perlovsky, L. I., Cabanac, A., Bonniot-Cabanac, M-C. \& Cabanac, M. Mozart effects, cognitive dissonance, and the pleasure of music. Behav Brain Res 244 9-14 (2013).

28. Stroop, J. R. Studies of interference in serial verbal reactions. J Exp Psych 18, 643-682 (1935)

29. Schroeter, M. L., Zysset, S., Wahl, M. M. \& von Cramon, D. Y. Prefrontal activation due to Stroop interference increase during development. NeuroImage 23, 1317-1325 (2004). 
30. Trainor, L. \& Heinmiller, B. M. The development of evaluative responses to music: infants prefer to listen to consonance over dissonance. Infant Behav Dev 21, 77-88 (1998).

31. Trainor, L., Tsung, C. D. \& Cheung, V. H. W. Preference for sensonry consonance in 2- to 4-month-old infants. Music Percep, 20, 187-194 (2002).

32. Masataka, N. Preference for consonance over dissonance by hearing newborns of their deaf parents and of hearing parents. Dev Sci 9, 46-50 (2006).

33. Zysset, S., Muller, K., Lohmann, G. \& von Cramon, D. Y. Color-word matching Stroop task: separating interference and response conflict. NeuroImage 13, 29-36 (2001).

34. Moser, S. J., Cutini, S., Weber, P. \& Schoreter, M. L. Right prefrontal brain activation due to Stroop interference is altered in attention-deficit hyperactivity disorder - a functional near-infrared spectroscopy study. Psychiat. Res :Neuroim 173, 190-195 (2009).

35. Zentner, M. \& Kagan, J. Infants' perception of consonance and dissonance in music. Infant Behav Dev 21, 483-492 (1998).

36. Lundin, R. W. An objective psychology of music, $3^{\text {rd }}$ edition (Krieger, 1985).

37. Frances, R. The perception of music (Erlbaum, 1988).

38. Perlovsky, L. I. Cognitive function, origin, and evolution of musical emotions. Music Sci 16, 185-199 (2012).

39. Huron, D. Why is sad music pleasurable?: a possible role for prolactin. Music Sci 15, 146-158 (2011).

40. Monsell, S., Taylor, T. J. \& Murphy, K. Naming the color of a word: is it responses or task sets that compete? Memory $\operatorname{Cog} 29,137-151$ (2001).

41. Adelman, N. E. et al. A developmental fMRI study of the Stroop color-word task. NeuroImage 16, 61-75 (2002).

42. Spieler, D. H., Balota, D. A. \& Faust, M. E. Stroop performance in healthy younger and older adults and in individuals with dementia of the Alzheimer's type. J Exp Psychol Hum Percept Perform 22, 461-479 (1996).

43. Derfuss, J., Brass, M., Neumann, I. \& von Cramon, D. Y. Involvement of the inferior frontal junction on cognitive control: meta-analysis of switching and Stroop studies. Hum Brain Mapp 25, 22-34 (2005).

44. Jennings, J. M., Dagenbach, D., Engle, C. M. \& Funke, L. J. Age-related changes and the attention network task: an examination of alerting, orienting, and executive function. Neuropsychol Dev Cogn B Aging Neuropsychol Cogn 14, 353-369 (2007)

45. Madden, D. J. \& Gottlob, L. R. Adult age differences in strategic and dynamic components of focusing visual attention. Aging Neuropsychol Cogn 4, 185-210 (1997).

\section{Acknowledgments}

This research was supported by a grant (\#25285201) as well as by the Grants for Excellent Graduate Schools, from the Ministry of Education, Science, Sports and Culture, Japanese Government (A06 to Kyoto University). We are grateful to Naoko Watanabe for assistance when conducting experimentation and Masahiro Shibasaki and Elizabeth Nakajima for making comments on an earlier version of this manuscript. We are also thankful to reviewers for suggestions.

\section{Author contributions}

N.M. and L.P. conceived the study, and participated in its design and coordination and drafted the manuscript. N.M. conducted the experiments and participated in the data analysis and interpretation. Both authors read and approved the final manuscript.

\section{Additional information}

Competing financial interests: The authors declare no competing financial interests.

How to cite this article: Masataka, N. \& Perlovsky, L. Cognitive interference can be mitigated by consonant music and facilitated by dissonant music. Sci. Rep. 3, 2028; DOI:10.1038/srep02028 (2013)

(c) (i) $(-)$ This work is licensed under a Creative Commons AttributionNonCommercial-NoDerivs 3.0 Unported license. To view a copy of this license, visit http://creativecommons.org/licenses/by-nc-nd/3.0 